Evidence for Multiple Strategies in Off-beat Tapping With Anisochronous Stimuli*

Jacques Launay, Roger T. Dean, Freya Bailes

MARCS Institute, University of Western Sydney

Author for correspondence:

Jacques Launay

Experimental Psychology

Tinbergen Building

South Parks Road

University of Oxford

Oxford

OX1 3UD

UK

Email: $\quad$ jacques.launay@psy.ox.ac.uk

Phone: $\quad$ (+44) 1865527238

* published in full as: Launay, J., Dean, R. T. \& Bailes, F. (2013) Evidence for multiple approaches to off-beat tapping with anisochronous stimuli. Psychological Research. doi: 10.1007/s00426-013-0513-9.

The final publication is available at http://link.springer.com/article/10.1007\%2Fs00426$\underline{013-0513-9}$ 


\begin{abstract}
There is a large body of evidence relating to the ways that people tap in time with sounds, and perform error correction in order to do this. However, off-beat tapping is less well investigated than on-beat tapping. The current study involves co-ordinating with a stimulus sequence with underlying isochrony and systematic deviations from this isochrony that increase or decrease in magnitude to look at people's capacity to error correct when performing off-beat synchronisation with a set of sounds. Participants were instructed to 'tap between the tones' but 'try to maintain regularity'. While analysis using typical methods suggested a form of error correction was occurring, a series of more complex analyses demonstrated that participants' performance during each trial can be classified according to one of four different strategies: maintaining a regular pulse, error correction, phase resetting, and negative error correction. While maintaining a regular pulse was the preferred strategy in conditions with increasingly isochronous stimuli, the majority of trials are best explained by other strategies, suggesting that participants were generally influenced by variability in the stimuli.
\end{abstract}




\section{$\underline{\text { Evidence for Multiple Strategies in Off-beat Tapping With Anisochronous Stimuli }}$}

For people who synchronise well with rhythmic sound the task appears to be one requiring little overt attention. However, extensive psychophysical experimentation and modelling reveal that our sensorimotor skills require monitoring and error correction processes in order to maintain accuracy when tapping along to even an isochronous pulse (for a review see Repp, 2005b). As these processes are thought to be largely automatic (e.g. Repp, 2002a), it is of interest to understand to what extent they can be modified if given instructions to do so, and how variable stimuli affect the error correction process. The current study uses anisochronic sequences to investigate how people adjust off-beat tapping in response to variation in a stimulus sequence when they have been instructed to maintain regularity. Anisochronic sequences were generated by jittering each stimulus in an isochronic sequence by a certain amount, that either increased or decreased throughout the course of the sequence. Participants were non-musicians and were untrained in tasks of this kind, so we expected their performance not to be influenced by the development of specific strategies to maintain an internally generated regular pulse.

While on-beat synchronisation with series of tones has been extensively studied, there is less evidence relating to the ways in which people perform off-beat synchronisation. This has the capacity to be an ambiguous task which is influenced by the instructions given and allows various phase-locks in order to develop a steady relationship with isochronous stimuli. For example, tapping exactly $200 \mathrm{~ms}$ after each beat of a $600 \mathrm{~ms}$ pulse is just as 'synchronised' as tapping $400 \mathrm{~ms}$ after the beat is heard. This ambiguity means asynchronies may be measured from a tone preceding or following 
a tap, although here we will always refer to asynchronies as the time from one tone to the following tap made. Anti-phase synchronisation (tapping exactly halfway between successive tones) could be considered the archetype of off-beat tapping, and from a dynamic systems perspective this degree of off-beat phase lock should be easiest to maintain (e.g. Pikovsky, Rosenblum, \& Kurths, 2001). Studies have shown that human performance demonstrates similar properties to dynamic systems models, with anti-phase synchronisation acting as a stable strategy, but with a tendency to revert to more stable on-beat synchrony (Amazeen, Schmidt, \& Turvey, 1995; Schmidt \& O'Brien, 1997).

The potential for off-beat tapping tasks to be influenced by instructions has previously been explored by Repp (2001), by giving specific instructions to tap just before the auditory beat, or just after it, which can give the subjective experience of 'leading' or 'following' the beat (Vos \& Helsper, 1992), and in Repp's study both appear to be less stable strategies (i.e. participants exhibit more problems maintaining a steady beat) than anti-phase tapping. The current experiment further exploited the potential for ambiguity by giving no instructions relating to the kind of phase lock required with the stimulus during off-beat tapping, but telling participants to try to maintain a regular beat during the trial. In this way we explored how people perform off-beat tapping when they do not have a prescribed temporal target, but may be distracted by deviation in the stimuli. With this ambiguity we probe whether people are more likely to maintain an internally generated regular beat, or whether their performance will track changes in the stimuli. This could not be so effectively evaluated using on-beat tapping, in which asynchronies between tone and tap times would act as markers of the variability in the tone sequence. 
It is often assumed that people normally correct performance in on-beat synchronisation according to differences between tone and tap time, and that most of this error correction occurs at the tap immediately following the asynchrony (Mates, 1994b; Michon, 1967; Vorberg \& Schulze, 2002; Vorberg \& Wing, 1996). There is evidence suggesting that the error correction response is a subconscious one (Repp, 2002a, 2002b), will occur when errors are subliminal (Repp, 2000, 2001), and even when there are specific instructions to ignore perturbations in the stimulus sequence (Repp, 2002a). While error correction is typically studied as the adjustment to an intertap interval (ITI) made immediately following the perception of an asynchrony, it is important to note that there is substantial evidence suggesting that sequences of asynchronies will exhibit positive autocorrelation (e.g. Michon, 1967; Pressing, 1998), with up to three lags having some predictive value on the measured asynchrony in the tapping sequence that people will tap with. Given that asynchronies and ITIs are dependent on one another this autocorrelation should be explicable by any model that is created to predict ITIs. Additionally, it should be noted that statistical models that do not account for this autocorrelation are not statistically legitimate.

A notable alternative to error correction as a strategy in sensorimotor synchronisation is a mixed phase resetting strategy (e.g. Hary \& Moore, 1987). This refers to performance that switches randomly between using the previous tap time and previous tone time as a reference point for the next tap. It has been argued that this strategy is a convincing explanation of many sensorimotor synchronisation results (Repp, 2005b) including off-beat tapping performance (Repp, 2004). 
With off-beat tapping, given the potential ambiguity as to when to tap in relation to a tone, it is less obvious that error correction should occur on the basis of differences between tap and tone times. Yet error correction does occur during off-beat tapping, and is comparable to on-beat tapping, with similar correction occurring while responding to isochronous stimuli (Y. Chen, Ding, \& Kelso, 2001; Pressing, 1998; Repp, 2002a) and at both supraliminal (Vos \& Helsper, 1992) and subliminal levels of deviation from isochrony (Repp, 2001) in stimulus sequences. These studies suggest that correction is complete (i.e. there is no long-term change in tapping behaviour), and occurs within the first few taps following an introduced perturbation, making it similar to that which occurs for on-beat tapping.

The asynchronies involved in on-beat and off-beat synchronisation are quite different however. Error correction to on-beat tapping requires monitoring of small asynchronies between tap and tone times, and small error correction on the basis of inaccuracies. For error correction during off-beat synchronisation, asynchronies between tap and tone times are greater than those during on-beat tapping, but error correction should still be small. As well as the discrepancy between asynchrony size and size of error correction required, there is an additional complication. Error correction becomes less accurate when asynchronies become large enough to be detected (Madison \& Merker, 2004), so error correction for off-beat synchronisation should be worse than that for on-beat synchrony (because asynchronies are larger, and more likely to be detectable). This has not been supported experimentally however, and error correction is equally effective during off-beat tapping as with on-beat (Pressing, 1998; Repp, 2001, 2002a). While mixed phase resetting may be a stable strategy in on-beat tapping, switching 
between using tap and tone times as a guide to tapping off-beat would require changing the interval after which tapping occurs, complicating the processes underlying the monitoring of this periodicity (i.e. when tapping off-beat to a $600 \mathrm{~ms}$ pulse, using the tap times as a guide to when to tap next would require monitoring an interval of approximately $600 \mathrm{~ms}$ before tapping, while using the tone times would require monitoring an interval of $300 \mathrm{~ms}$, so switching between these two seems an unlikely strategy). In the current study, by minimising purposeful participant attention to continuous perturbations and therefore conscious error correction, we test whether correction will occur and in what manner.

While similar off-beat correction has been reported to on-beat correction, there is recent evidence to suggest that the type of paradigm used to evoke error correction can act as a significant influence on the results (Repp, Keller, \& Jacoby, 2012). Single perturbation studies, in which participants tap along with an isochronous stimulus and one deviation is introduced, tend to give higher estimates for error correction than methods with changes occurring at each interval in the stimulus set (introduced using an adaptive timer modelled on human behaviour) or methods using isochronous sequences. The current study uses variable stimulus intervals that change throughout a trial because this relates closely to variability in human rhythms that are not computer generated. Instead of participants monitoring stimuli for one unusual event or correcting just their own errors in response to an isochronous sequence they might perform continuous error correction relating to their own performance and the stimuli throughout trials. Participants were instructed to maintain regularity so they should attempt to minimise distraction provided by variability in the stimuli, but previous evidence shows that people 
will be affected by stimulus irregularity even trying to ignore this irregularity (Kato \& Konishi, 2006; Repp, 2006; Repp \& Penel, 2004).

Expertise is also likely to influence the extent to which error correction will occur, and people with no musical expertise show greater variability in intertap intervals than those with musical expertise, and worse error correction when aware of stimulus deviation (J. L. Chen, Penhune, \& Zatorre, 2008; Drake, Penel, \& Bigand, 2000; Madison \& Merker, 2004; Pressing, 1998). However, while musical expertise may improve performance generally, repeated exposure to tasks of this kind (i.e. specific error correction expertise rather than musical expertise) can result in improved tapping regularity and lower levels of error correction (Repp, 2010). In the current study we tested participants who are not musical experts, as assessed by a questionnaire, the Ollen Musical Sophistication Index, (OMSI; Ollen, 1996), and have little experience with tasks of this kind. The OMSI is a sensitive test of musical expertise, which takes into account a number of features of musical sophistication, including prior musical training, current involvement and general interest, to give a probability figure as to whether someone should be classified as a musician; OMSI values of less than 500 suggest less than a $50 \%$ probability that a person is a musician, and imply less than two years of musical training (Ollen, 1996).

Using participants who are not familiar with tapping experiments and are not given the opportunity to practice over many trials means we are measuring error correction responses that have not been honed. In this way we can assess whether people are naturally drawn to external stimuli as timekeeping mechanisms, or more likely to use their own internal processes to maintain a regular pulse. In order to ensure that these non- 
expert participants were capable of performing the tapping task, a practice procedure was developed, which required a minimum standard of performance in order for participants to progress to the experiment.

Given the potential for different forms of off-beat tapping behaviour, we have considered several possible outcomes (see Figure 1 for examples) which cover the range of plausible ways in which people might perform. These 'strategies' (by which we simply mean behaviour adopted) are the following:

1. A 'regular pulse' will involve no error correction, and demonstrate that participants were able to maintain regular tapping, despite distraction from continuous and unpredictable changes in the stimuli. Figure 1 demonstrates that in this case there will be a relationship between IOIs and asynchronies, but that variability in IOIs will not relate to any adjustment of ITIs, which remain constant.

2. 'Error correction' will involve conventional error correction, in which intertap intervals are adjusted by some approximately constant amount according to the previous asynchrony. The proportion of correction based on the previous asynchrony may vary between 0 and 1, with larger amounts suggesting close to perfect error correction. In Figure 1, ITIs in this case have been calculated using 'perfect' error correction (i.e. with a coefficient of 1).

3. An alternative strategy, 'phase resetting', involves tapping with a constant asynchrony between tones and taps. Tapping a constant delay after each tone would imply that participants are adjusting tap times to maintain this 
asynchrony, rather than monitoring the time between adjacent taps, and that the reference point for timing asynchronies is updated when they hear each tone. This strategy would approximate error correction, but makes different predictions about performance, as shown in Figure 1. While it could be characterised using some error correction constant, the more pertinent feature of this performance is that it involves a constant asynchrony (so asynchronies do not correlate with IOIs), which can be assessed separately from the estimation of error correction constants. It relates to mixed phase resetting, but does not include reference to the previous tap time, which would involve switching the period that is monitored before making a tap.

The current study investigates whether, in addition to correction during off-beat tapping occurring with perturbations, error correction in off-beat tapping occurs even when the instructions given oppose this behaviour (Repp, 2002b). As previous research has suggested that people identify sequences with up to $4 \%$ deviation from isochrony as isochronous, but only begin to lose the feeling of a regular underlying beat when there is $8.5 \%$ deviation from isochrony (Madison \& Merker, 2002), these thresholds were included in the range of anisochrony used in order to test whether the thresholds affected the level of error correction in the current paradigm.

No explicit instructions were given regarding the kind of phase lock that participants should make with the stimuli. They were instructed to tap at a different time from the tones, make one tap for each tone heard, and try to maintain a regular pulse. As 
the stimuli were anisochronous, with continuous deviations from an underlying pulse, the instruction to maintain a regular pulse would require participants to ignore these changes in the stimuli, and avoid distraction. To reiterate, we would expect the current experimental paradigm to minimise the intentional monitoring of asynchronies, both because of the instructions and participants involved. The aim was to test whether an error correction response would still exist in this paradigm and we expected this to be the case, in line with previous literature arguing that the error correction response occurs even when perturbations are very small, or when participants are told to ignore changes.

\section{Method}

\section{Participants}

Data from 34 staff and students from the University of Western Sydney who scored below 500 on the OMSI were analysed (mean age $=21.9, \mathrm{SD}=5.6$; mean OMSI $=132.8, \mathrm{SD}=88$, male $=10)$. The experiment was approved by the University of Western Sydney Ethics Committee.

\section{Materials}

Participants heard tones over Sennheiser HD 650 headphones (played via an external Edirol UA-25 Soundcard) and responded using a Roland Handsonic HPD-10 drum pad. The tones played were a synthesised woodblock sound with an attack time of 2 ms and a decay time of $10 \mathrm{~ms}$. The whole experiment, including the practice, was controlled and sequenced using MAX/MSP v. 5.0.8. 
The series of tones played during tapping trials was an anisochronic sequence, constructed as in Madison and Merker (2004). Each IOI was either shortened or lengthened by a fixed interval from an underlying IOI of $600 \mathrm{~ms}$. Shortening or lengthening was determined by a Kolakoski sequence (Kolakoski \& Ucoluk, 1966); this binary sequence would be unpredictable to listeners, but contains a roughly equal number of ones and zeros, and allows neither of these to be repeated more than twice in a row. For each zero in the sequence a shortened interval would be used to determine the time of the next tone, and for each one, a lengthened interval would be used. In both conditions the level of anisochrony started at $45 \mathrm{~ms}$, meaning a shortened interval would be $555 \mathrm{~ms}$, and a lengthened one would be $645 \mathrm{~ms}$. Throughout the trial this changed by $22.5 \mathrm{~ms}$ every 32 taps, and either increased anisochrony (i.e. changing intervals to $532.5 \mathrm{~ms}$, and $667.5 \mathrm{~ms}$ then $510 \mathrm{~ms}$ and $690 \mathrm{~ms}$ ) or decreased anisochrony (making intervals of 577.5 $\mathrm{ms}$ and $622.5 \mathrm{~ms}$, then an isochronous pulse of $600 \mathrm{~ms}$ ). The IOI sequence demonstrates autocorrelation of approximately - 0.2 and as it was the same for all participants this autocorrelation should not have any influence on individual variability in response, which is our focus here.

\section{Design}

Here we report results from two experimental conditions, in which stimuli either became or increasingly anisochronous or decreasingly anisochronous throughout the trial. Tone and tap time data were recorded, and the tap time data are the dependent variables.

\section{Procedure}


These data were collected as part of another virtual interaction experiment, in which participants would also play an economic game with a computer tapping partner, and results relating to that are reported elsewhere without any detailed modelling of tapping performance (Launay, Dean, \& Bailes, 2013). Participants were told the experiment was about rhythmic games, and how they would interact with a series of partners, and all gave written informed consent before the experiment began.

Practice in tapping out of time with stimuli and a condition in which participants were required to synchronise (not reported here) was given at the start of the experiment. In these practices visual feedback was given for each tap made to indicate whether participants had tapped correctly or incorrectly, and only allowed participants to progress to the next practice trial after they had made enough correct taps in relation to incorrect taps (50 correct taps after subtracting incorrect taps). In the practice, four consecutive trials of isochronous beats were played over headphones, each one slightly faster than the previous, with increasingly stringent accuracy criteria (reaching a final interonset interval of $600 \mathrm{~ms}$ with "correct" taps determined by a $100 \mathrm{~ms}$ window around the stimulus onset). At the start of the experiment, all participants performed a "synchronise" version of this, followed by a “don't synchronise" version, during which correct responses were judged by whether tapping was outside a $100 \mathrm{~ms}$ window around the tone onset. The final trial of this practice was repeated before each of the real tapping trials, preparing participants for the ensuing tapping trial by giving them a practice of the same kind as they were about to perform (i.e. "synchronise" or "don't synchronise"), and requiring their performance to be of a good enough standard before they could progress. 
In the part of the experiment analysed here, participants heard tones and were given the instruction not to synchronise with the tones by tapping on the drumpad. It was explained that "don't synchronise" meant they should "aim to tap at a different time from the tones" they were hearing, but try to maintain regularity and make one tap for each tone they heard. This instruction relating to tapping a regular beat was given three times: twice in the instruction sheet participants read before the study ("ALWAYS try to monitor your tapping to beat as regularly as possible on the drum pad", and "ALWAYS try to tap a regular beat on the drum"), and was also present during the tapping experiment in the bottom right hand side of the screen ("ALWAYS try to tap a regular beat"). Two different trials were collected for each participant, one with increasing isochrony in the stimulus sequence, and the other with increasing anisochrony, and the order of trials was counterbalanced. The tapping part of the experiment analysed here lasted approximately 5 minutes.

During the tapping task, participants were given no feedback, and could not hear their own taps, apart from the gentle thud of their finger on the drum pad. Each trial ran until the participant had made 106 taps in total, and the IOI of tones were determined by one of two strategies employed by the computer system, becoming more anisochronous in one trial and less anisochronous in the other trial. The first 10 taps made by each participant were not recorded by the system, as these might exhibit high variability as participants adjusted to the task.

\section{Results}


The data were analysed in two different ways using the statistics program R: one used typical pooled analysis to look directly at the relationship between IOI and timing of the following tap made, and used exclusion criteria to omit taps that did not fit usual patterns (e.g. if a participant tapped twice for one tone). The second approach used a set of more complex analyses to better investigate individual variability in behaviour. Time series modelling was performed on individual data sets, demonstrating considerable differences between individuals, so data were grouped according to strategies described in the introduction in order to perform multilevel linear modelling on smaller groups of participants. The method used for grouping participant data is described later in this section.

Histograms of pooled raw data for ITIs and asynchronies are given in Figure 2. The histograms for ITIs exclude values greater than $1500 \mathrm{~ms}$ to make them easier to interpret (23 data points excluded in the decreasing anisochrony condition, and 21 excluded in the increasing anisochrony condition). Averaging ITIs and asynchronies within each trial then across participants gives a mean ITI in the increasing anisochrony condition of $652 \mathrm{~ms}(\mathrm{SD}=134 \mathrm{~ms})$ and $616 \mathrm{~ms}(\mathrm{SD}=126 \mathrm{~ms})$ in the decreasing anisochrony condition, demonstrating that participants generally tapped at approximately the expected tapping rate $(600 \mathrm{~ms})$, although were prone to longer ITIs in the increasing anisochrony condition. Mean asynchronies in the increasing anisochrony condition were $302 \mathrm{~ms}(\mathrm{SD}=40 \mathrm{~ms})$ and $307 \mathrm{~ms}(\mathrm{SD}=43 \mathrm{~ms})$ in the decreasing anisochrony condition, which suggest that participants did generally tap quite close to antiphase with stimuli. In total, 444 taps (out of 3264) were missed in the increasing anisochrony condition, and 288 taps were missed in the decreasing anisochrony condition. These relatively high 
numbers of missed taps are likely to be a consequence of using participants who did not have any expertise in tasks of this kind, and must be taken into account in statistical analysis.

\section{Pooled analysis}

Timing of taps and tones were first compared and each tap was paired to the previous tone heard. Asynchronies are therefore all presented as positive values, and represent the distance of the current tap from the last tone heard. Increasing anisochrony and decreasing anisochrony trials were analysed together, and in the same manner. Data were first assessed to determine whether participants had successfully followed the instruction to produce one tap for each tone heard. ITIs that were greater than $900 \mathrm{~ms}$ were excluded from analysis of intertap intervals as they would indicate a missed tone. ITIs less than $300 \mathrm{~ms}$ were also excluded because they would indicate participants tapping more than once for each tone ( $\sim 9 \%$ of the data were excluded on this basis). For asynchronies, as there was no instruction about when participants should tap in relation to the tone, separate exclusion criteria were used, with outliers excluded on the basis of being more than 2 standard deviations from the trial mean $(\sim 4 \%$ of the asynchrony data were excluded on this basis).

In order to look at tapping rate variability within each trial, each remaining ITI data point was normalised in relation to the trial mean. Mean ITIs were calculated for each trial, and each ITI was then recalculated as a deviation from the trial mean. This removes variability in mean tapping rate across participants, allowing pooling of the data. 
This was done similarly for asynchronies, and is more important for this measure, given that participants could tap at quite different times after each tone heard.

The above normalisation calculations gave ITIs and asynchronies relative to the trial mean at each time point, which can be compared with the preceding IOIs. The relationship between these two can reveal how people's tapping was affected by the most recent stimulus. Figure 3 gives means for ITI deviations and asynchrony deviations following each IOI (outliers more than 2 standard deviations have been excluded at each IOI, approximately an additional $10 \%$ of data points). After exclusion of outliers there is a positive linear relationship between IOI and following ITI (linear model intercept $=-5.0$ s.e. $0.61 \mathrm{p}<0.0001$, slope $=0.35$, s.e. $0.01 \mathrm{p}<0.0001$, Adj $\mathrm{R}^{2}=0.13, \mathrm{p}<0.0001, d f=$ 5986), and a negative relationship between IOI and asynchrony (linear model intercept $=$ 5.6 s.e. $1.1 \mathrm{p}<0.0001$, slope $=-0.13$, s.e. $0.02 \mathrm{p}<0.0001, \mathrm{Adj}^{2}=0.006, \mathrm{p}<0.0001, d f$ $=6127)$. However, this linear trend does not explain performance during the final third of the decreasingly anisochronous sequence, when there is no variability in the stimulus sequence (i.e. when people are performing with an isochronous sequence). This suggests that the constant period in the decreasing anisochrony condition did not involve the same kind of tapping behaviour that occurred in the variable stimulus periods.

The difference in slope, and adjusted $\mathrm{R}^{2}$ values of the models for asynchrony and ITI measures suggests a large discrepancy between the amount of variability that is explained by the previous IOI for these two measures, meaning that this model does not include a sufficient predictor of asynchronies. This discrepancy is likely to indicate phase resetting, which, as demonstrated in Figure 1, will involve a good relationship between IOIs and ITIs but not between IOIs and asynchronies. However, given that there is a 
significant relationship between IOIs and asynchronies, this result could also represent error correction, or alternatively be a consequence of the group average being unrepresentative of individual patterns of behaviour. Further analysis, involving modelling of individual variability in response might help to explain why IOIs are not as good at predicting the following asynchronies as they are at predicting ITIs.

Given that these relationships could be affected by whether the anisochrony in the stimulus was detectable or not, they were recalculated for each level of anisochrony. Linear relationships were calculated as above for each of the anisochrony levels: either $3.75 \%$ (IOIs of $577.5 \mathrm{~ms}$ and $622.5 \mathrm{~ms}$ ), $7.5 \%$ (IOIs of $555 \mathrm{~ms}$ and $645 \mathrm{~ms}$ ), $11.25 \%$ (IOIs of $532.5 \mathrm{~ms}$ and $667.5 \mathrm{~ms}$ ), or $15 \%$ (IOIs of $510 \mathrm{~ms}$ and $690 \mathrm{~ms}$ ). The calculated coefficients are given in Figure 4 (after exclusion of outliers at each level). These do not appear to demonstrate any systematic differences in performance associated with the different levels of anisochrony. There is no obvious trend relating to greater or smaller amounts of anisochrony in the stimuli, and no obvious changes associated with the thresholds associated with awareness of anisochrony (4\% and $8.5 \%$ ).

\section{Analysis of individual differences}

The first form of analysis revealed some ambiguity in the dominant form of tapping behaviour. The relationship between IOIs and ITIs suggests phase resetting is occurring, but if this were the only strategy occurring then we would expect no relationship between IOIs and asynchronies, and this was not the case. Instead, it seems likely that this strategy explains some participant data, but not all, so results were also analysed in a different manner, modelling all of the asynchronies and ITIs using 
preceding tap and tone time information (instead of excluding double taps, taps following missed tones, and outliers) using time series analysis. Tap and tone data were matched as before, and the increasing anisochrony and decreasing anisochrony conditions were analysed separately meaning each data set had 96 data points. Coding was added using a one or zero for each tap to indicate whether a preceding tap had been missed (i.e. if there had been a tone that had no tap attached), and separate coding if a 'double' tap had occurred (i.e. participants had tapped more than once to the corresponding tone). The number of taps missed since the last tap made were also coded as a separate variable where this was greater than zero. The level of anisochrony in the stimulus (either $7.5 \%$, $3.75 \%$ or 0 in the increasing isochrony condition and $7.5 \%, 11.25 \%$ or $15 \%$ in the increasing anisochrony condition) was also coded for each tap, with the three levels labelled as 1, 2 or 3 within each trial. Single ITIs that were longer than $3000 \mathrm{~ms}$ (i.e. participants had missed more than five successive taps) were excluded from analysis as these caused skewing of the models produced (four data points were excluded on this basis in total which can be compared with the much larger proportion of data points excluded in the pooled analysis).

It was thought that the tapping series were likely to exhibit autocorrelation (i.e. successive asynchronies, and ITIs would have some predictive value on one another), as has previously been found (e.g. Michon, 1967; Michon \& Van der Valk, 1967; Pressing, 1998) and that this should be modelled. In order to confirm this, and confirm the number of lags that needed to be modelled in order to address autocorrelation, time series analysis was first performed on individual sets of data. Models were only created for the asynchrony measure, because this more complex form of analysis can reveal sufficient 
detail about tapping behaviour given that ITIs are a linear combination of differences between successive asynchronies and IOIs.

Three data sets were picked at random in the decreasing anisochrony condition and the best time series models were determined for each individual data set using backward stepwise modelling. This analysis was done to give an indication of what predictors might be important for time series models performed on all data. Each model started with the following predictors: three autoregressive (AR) components (lag 1-3), previous IOI, coding for whether a tap had previously been missed, the number of taps missed, coding for whether a double tap had occurred, and coding for the level of anisochrony. Components were removed from the model if they did not act as significant predictors, or if their removal improved the Bayesian Information Criterion (BIC), suggesting a similarly good, but more parsimonious model. Residuals were assessed for remaining autocorrelation, and were also tested using Bartlett's periodogram-based test to ensure they were white noise. Moving average components which would model relationships across greater distances in the series were not included in any models as these would not yield readily interpretable results. The results are given here purely as a demonstration of large variability in necessary model components, and coefficients of these components. Coefficients of autoregression in all results reported are the coefficient for autoregression of the residual error following linear modelling of the asynchrony. The raw ITI data for these three examples have been given in Figure 5.

Data from the first individual to be modelled in the decreasing anisochrony condition is shown in the top panel of Figure 5, and raw data suggests that these ITIs were generally well matched to the IOI sequence, with ITIs correlating well with the 
previous IOI, except in one case where a tap has been missed. This missed tap should be effectively modelled by including a coefficient for missed taps. The asynchronies initially had a non-stationary series as determined by an augmented Dickey-Fuller test (Said \& Dickey, 1984), which means that the asynchrony changed over the series, and cannot be statistically modelled without transformation. The asynchrony and previous IOI were therefore both first differenced (subtracting successive values from one another), giving a stationary series. This makes time series analysis statistically legitimate, but makes interpretation of final coefficients less straightforward than using an untransformed series, so results should be interpreted with caution. The best model for this individual included the differenced previous IOI (differenced IOI coefficient: -0.20 , se $=0.064, p=$ 0.0017), two autoregressive components (AR1: -0.35 , se $=0.11, \mathrm{p}=0.0015$; AR2: -0.27 , $\mathrm{se}=0.10, \mathrm{p}=0.008)$ and coding for the number of taps missed since the last tap made (number of taps missed coefficient: -167.8 , se $=22.1, \mathrm{p}<0.0001$ ). This coefficient for missed taps suggests that after the missed tap, the asynchrony was $167.8 \mathrm{~ms}$ shorter than if the participant had not missed a tap (i.e. after missing a tap, the participant tended to tap too early after the following tone). Coefficients for missed and double taps are primarily included because they allow all data points to be included in analysis, rather than excluding outliers (such as the large ITI that occurs once in this sequence).

The next randomly selected individual had a large number of missed taps in the data set (indicated by the outlying large ITIs in the second panel of Figure 5) but an augmented Dickey-Fuller test suggested that the asynchrony series was stationary. The best model included previous IOI (IOI coefficient: 0.80 , se $=0.049, \mathrm{p}<0.0001$ ), one AR component (AR1: 0.61, se $=0.08, \mathrm{p}<0.0001)$, along with coding for missed taps (missed 
taps coefficient: -280.8 , se $=37.4, \mathrm{p}<0.0001$ ) and double taps (double taps coefficient: 215.1, $\mathrm{se}=39.7, \mathrm{p}<0.0001)$. The coefficient for a double tap suggests that second taps made after a tone had on average an asynchrony $215.1 \mathrm{~ms}$ greater than that of taps made immediately following a tone.

The final data set (third panel in Figure 5) had quite poorly matched ITIs and IOIs, with much larger variability in tapping rate compared with that in the stimulus sequence. The asynchronies and IOIs again required first differencing in order to be stationary. The best model included three AR components (AR1: -0.52 , se $=0.10, \mathrm{p}<$ 0.0001; AR2: -0.38, se $=0.11, \mathrm{p}=0.0003 ;$ AR3: $-0.28, \mathrm{se}=0.10, \mathrm{p}=0.0048)$, and coding for double taps (double taps coefficient: 444.5, se $=47.6, \mathrm{p}<0.0001$ ) and missed taps (missed taps coefficient: -467 , se $=50.5, \mathrm{p}<0.0001$ ).

Conclusions from this analysis were that coding for the level of anisochrony did not tend to improve models, but variability between individual models was high. Analysing all trials together as in the pooled analysis might therefore conflate differences, for example in the relative importance of different AR components, or in the previous IOI. In addition to performing full time series analysis on these few data sets, all data sets were also analysed using automated time series analysis, to determine the number of AR components that might be relevant in modelling the tapping behaviour of different participants, and the coefficients of these. These models only assessed autocorrelation (i.e. no other predictors were included), all used first differenced asynchronies, and used the function 'auto.arima' provided in the 'forecast' package version 2.12 for $\mathrm{R}$, using BIC to determine the best model. Summary statistics for these coefficients are given in Table 1 . This modelling means we can assess the number of AR 
components that are relevant to different participants; these demonstrate that at least one AR component should be modelled in almost every case, two AR components were relevant for the majority of trials, and up to five could have some predictive value on asynchronies.

Given large variability in individual models, we took each trial separately and modelled each trial according to the distinct types of performance outlined in the introduction in order to determine which best fitted the data - maintaining a regular pulse (i.e. no error correction), error correction, and phase resetting. While there could be some flexibility in strategy throughout a trial, by modelling each trial using one of these three types of behaviour, and using maximum likelihood to determine which of these models gave the best fit for the data, we can more effectively group data sets according to strategy. For this analysis, asynchronies following double taps and missed taps were excluded, because these could undermine the robustness of the fairly crude modelling technique. This meant the exclusion of two participants in the increasing isochrony condition, and two in the increasing anisochrony condition, because they consistently made mistakes in the trial, either tapping more than once for a tone or missing taps.

First, each trial was assessed to determine the error correction constant (between 0 and 1) that would best fit the data. This constant indicates the adjustment made to an ITI on the basis of the previous asynchrony (i.e. it is a constant multiplied by the previous asynchrony to give the adjustment made to the ITI). This was estimated by creating models of the data, by first taking each measured tap time. The median ITI for a trial was then added to the tap time, to give an estimate of when a participant might be expected to tap next. In order to calculate the error correction for that tap, 300 (half the median IOI) 
was subtracted from the previous asynchrony, and this value was multiplied by the error correction constant. This error correction value was then added to the expected tap time. This gave a modelled data set using AR1 error correction which could be compared with the recorded data, in order to determine how accurately it matched performance. A series of different models of this kind were created with different error correction constants, and maximum likelihood was estimated using Huber m-estimates (1964) of the squared residuals, to determine which error constant led to the best model. Using Huber mestimates of the residuals is a robust way to determine the proximity of observed to predicted data, and was used because large outlying values when participants performed in unexpected ways could skew the data without this robust estimation. The best constant was determined by varying the constant value, in order to minimise this likelihood estimate, using the 'optim' function in the statistics package R, with the limited-BroydenFletcher-Goldfarb-Shanno estimation algorithm with box constraints (L-BFGS-B) to limit the estimate to values between 0 and 1 . Different error correction constants were tested using this nonlinear optimisation problem solving equation in order to determine which constant would give the lowest residuals (as measured using Huber m-estimates). As a large number of participants demonstrated error correction constants of 0 , the constraints were widened, to include all values from -1 to 1 , meaning negative error correction could also be modelled, a strategy that had not been anticipated.

After determining the best error correction constants for each trial, a second step involved data simulation for each trial assuming that participants were repeating the same asynchrony after each tone heard (taken as the median asynchrony of each trial), i.e. including no error correction in the model. Maximum likelihood was again estimated 
using Huber m-estimates for the squared residuals. These maximum likelihood values were then compared with those for the model including error correction, and the model with the lowest value was taken as the best predictor of behaviour in each trial. Each trial in both increasing isochrony and increasing anisochrony conditions was therefore classified as exhibiting one of four strategies, including the three outlined in the introduction, and an additional, unexpected strategy:

1. Regular pulse: characterised by error correction constant close to zero.

2. Error correction: characterised by a positive error correction constant.

3. Phase resetting: characterised by lower maximum likelihood in the model using tapping with a regular asynchrony.

4. "Negative" error correction: characterised by a negative error correction constant.

Numbers of participants in each of these groups are given in Table 2. As each participant performed in both increasing isochrony and increasing anisochrony trials, we can also assess the degree of overlap between their types of behaviour in these different conditions and this is also given in Table 2. This demonstrates that participants were more likely to tap regularly in the condition with decreasing anisochrony, and exhibited most negative correction in the condition with increasing anisochrony (arguably the hardest condition to perform). The phase resetting strategy exhibited most consistency for participants between conditions. 
These eight groups were analysed separately using multilevel linear models with an AR3 correlation structure to predict the asynchrony. Multilevel linear modelling techniques (e.g. Gelman \& Hill, 2007) allowed each individual to be assigned different coefficients for predictors, meaning that the trial data for each individual was not assumed to be independent. The package 'nlme' version 3.1-97 was used for this analysis, with the function 'Ime', which allows the inclusion of autoregressive coefficients as well as simple nesting of data. All data was first differenced for this analysis, because some trials exhibited stationarity while others did not. From the time series analysis of individuals, three lags were considered to be sufficient in the autocorrelation sequence in order to successfully model the time series. Previous IOI, coding for double taps and missed taps, and the number of missed taps were also included in models for each group within each condition. As in individual models, coding for the level of anisochrony was also included in the models, but this did not improve them, and did not demonstrate any systematic differences in behaviour, so the predictor was removed. AR coefficients for predictors in each model are given in Table 3. The purpose of these models was to indicate differences between the groups, so all groups have been modelled using the same components. While measures of the significance of AR coefficients cannot be estimated from the coefficients given using the 'lme' function, changing the number of AR coefficients for different groups would make it inappropriate to compare coefficients between different groups. These models are therefore not necessarily optimal for each of the different groups, but do make it possible to directly compare the relative influence of different factors in predicting asynchronies between groups. Given that these models were based on first differenced data, coefficients should 
be interpreted with some caution, particularly with regard to the autoregressive components, which cannot be taken as an indicator of any straightforward relationship between measurements.

AR coefficients are lower than those calculated for the models that do not include other predictors (given in Table 2). This suggests that including the IOI explains some of the variance that can also be explained by the autocorrelation of asynchronies. Results demonstrate relatively low levels of autocorrelation for participants who were tapping regularly. Participants with a phase resetting strategy appear to have high levels of autocorrelation between asynchronies in both conditions, suggesting that asynchronies in these cases were highly dependent on one another. Other strategies do not have consistent patterns between different conditions. Modelling double taps was important for almost all strategies in both conditions, indicating that asynchronies were significantly larger when double taps occurred. Similarly modelling missed taps was significant for all of the strategies and indicate that after a missed tap, asynchronies are smaller than normal, but as the number of missed taps had positive coefficients this earlier tapping after missing a tap became less substantial once several had been missed.

\section{Discussion}

The current results suggest that tappers with minimal specific training, and little musical expertise performed in a variety of ways when told to tap off-beat to anisochronous stimuli. Approximately a third of participants performed as they were instructed, and maintained a steady pulse, but this proportion was considerably lower in 
the conditions when they were tapping to increasingly anisochronous stimuli. Other participants appeared to perform according to three strategies, either using normal error correction, tapping with a regular asynchrony, or with a form of negative error correction. The results from grouping participants according to these strategies suggest distinct patterns of autocorrelation, but these were not consistent across decreasing anisochrony and increasing anisochrony conditions. The influence of stimuli applicable in all of the cases where participants did not maintain a regular ITI can be described as unconscious because it occurred at all levels of perturbation (indicated by pooled analysis), independent of whether they were at detectable levels, and also occurred despite the specific instructions given.

Using the ITI as a dependent variable in the first set of analyses showed that on average people modified their tapping rate according to the previous stimulus heard, with shorter ITIs following shorter IOIs. The ITI measure shows a comparable level of error correction to that of previous studies (e.g. Pressing (1998) reports a correction rate of .28 between successive asynchronies for a non-expert tapping off-beat to a sequence with an IOI of $750 \mathrm{~ms}$, compared with .35 suggested by the current data as calculated by the pooled analysis). However, while we would expect the change in asynchrony caused by each IOI to be similar, it is much smaller. The effect size in the best models for asynchronies is also smaller, suggesting that there is a larger amount of variability in the responses than that which is explained by the previous IOI. This could be interpreted as meaning that the asynchronies were relatively unaffected by the variation in the stimulus, which is hard to reconcile with the fairly large changes demonstrated in the ITI without further explanation. 
While we would expect the relationship between IOI and asynchrony to be of a similar magnitude to the relationship between IOI and ITI if participants were truly error correcting, this is not expected if they are performing with a regular asynchrony. The discrepancy is therefore better explained using the second set of analyses, which suggests that some participants are error correcting while others are not. The potentially distinct forms of behaviour identified are given some further explanation here.

\section{Regular Pulse}

Tapping with a regular ITI suggests that participants were performing as they were instructed. However, in order to do so, they would have to be ignoring the perturbations in the stimuli, resisting the tendency to error correct, and effectively avoiding synchronisation with the stimuli. This is in conflict with previous results, which have suggested that people will perform some error correction even when told not to do so (Repp, 2002b). Fewer participants were in this category in the condition with increasing anisochrony compared with the condition with decreasing anisochrony, suggesting that as changes in the stimuli became larger, the changes became more distracting, making it harder to ignore the variability as has previously been shown (Kato \& Konishi, 2006). The large number of participants in this group in the condition with decreasing anisochrony could be because the last third of the trial is isochronous, and likely to elicit fairly regular tapping. However, excluding this isochronous third of the data from the modelling analysis did not change the number of people for whom the best model was tapping with a regular pulse.

Multilevel linear modeling results demonstrated low levels of autocorrelation in 
the asynchrony series for this group, suggesting that this strategy of regular tapping led to less influence between subsequent taps. This would suggest that people performing in this way did so with an established tapping rate, that was relatively unperturbed by local variability in performance.

\section{Error Correction}

A number of participants performed error correction as we predicted. Previous papers attempting to model how people adjust during off-beat tapping have not suggested any major differences from models of on-beat synchronisation (Delignières, Torre, \& Lemoine, 2009; Pressing, 1998), and this appears to fit with the performance of approximately a third of our participants. These models suppose that the process of tapping off-beat is largely equivalent to that made when synchronising on-beat, with monitoring made of asynchronies, and corrections based on these. Some form of internal clock is required in order to perform this monitoring, and to trigger the next response (Mates, 1994a). However, while both Pressing and Delignieres suggest that tappers measure this asynchrony, and use this information to adjust the ITI, this must be at least somewhat modified during off-beat tapping, because asynchronies between tone and tap times are perceived at a different time in relation to when correction must be made.

\section{Phase Resetting}

The third group of participants used an alternative strategy to adjust ITIs. Instead of monitoring asynchronies and using these as a guide to modify the ITI, people may simply decide a distance from the current tone to tap, and attempt to maintain this as a 
constant interval. This would, in effect, adjust ITIs to the stimulus sequence, causing some degree of error correction without monitoring variability in the stimuli, instead simply resetting an internal time-keeper each time a tone is detected. The current results demonstrate how this process of phase resetting can be interpreted as an error correction response, as it does involve changes to ITIs of the same magnitude to perturbation in IOIs but in the current experiment, modelling demonstrates that behaviour is more parsimoniously explained without reference to error correction per se. There is already evidence that phase resetting might be a strategy employed during off-beat tapping ( Keller \& Repp, 2005), although that experiment, using musically trained participants and metrically organised stimuli, only demonstrated phase resetting at musically salient divisions of the synchronisation sequence. In experiments using less irregular stimuli, mixed phase resetting can similarly demonstrate a pattern that approximates error correction and is formally inseparable from this strategy.

The idea that tapping off-beat involves subdividing the interval between stimuli has previously been supported experimentally with evidence suggesting that the upper and lower values of IOI that people are capable of tapping off-beat with are roughly twice those of on-beat synchronisation (Fraisse \& Ehrlich, 1955; Repp, 2005a). This supports the argument that people subdivide the beat in order to tap off-beat (e.g. instead of timing $600 \mathrm{~ms}$ between taps, they time $300 \mathrm{~ms}$ from each tone to the following tap). If subdivision occurs when tapping off-beat it is unsurprisingly that the performance of many participants was best explained by a strategy of maintaining a regular asynchrony of approximately half of the stimulus IOI throughout trials. High levels of autocorrelation in participant data using this strategy indicate that local imperfections in asynchronies 
had more influence on tapping performance than that of participants tapping with a regular pulse. Participants tapping with a constant asynchrony are therefore more likely to experience drift throughout a trial, and are less able to maintain good timing throughout performance.

Within the group using phase resetting there appeared to be highest agreement between the two trial types, with almost all of the participants who performed in this way during the increasing anisochrony trial having performed similarly in the increasing anisochrony trial. It is also worth noting that all 4 participants who completed the experiment but were excluded from the present analysis because they were deemed to be musicians (with scores greater than 500 on the OMSI) also performed using this strategy. It is possible that this kind of performance is particularly stable, but this should be investigated further.

\section{"Negative" Error Correction}

A final group of participants performed in an unpredicted way, with adjustments made to their ITIs in the opposite direction to that predicted by normal error correction. This group was particularly large in the condition with increasing anisochrony, suggesting that as variability in the stimuli became more obvious, people resisted normal error correction processes more than before. This might indicate that they were making more of an effort to follow the instructions given, and maintain a regular ITI, resulting in exaggeration of this behaviour. Performance in these cases would not be synchronised with the stimuli, but the negative correction would help compensate for the increasing complication of co-ordinating with the stimuli. 


\section{Additional Conclusions}

A contentious issue within the literature on event timing is whether oscillator or discrete timer models are better at explaining how humans time events. Oscillator models are generally more biologically plausible and there is experimental evidence to suggest this may explain how synchronisation occurs (Large, 2000; Large \& Jones, 1999; Large \& Snyder, 2009). However, research on phase-resetting addresses 'interval' type models of timing. While this study was not designed to distinguish between oscillator and interval models of timing, an oscillator model of timing would most likely predict that participants would maintain a regular beat (as instructed), because this would involve simple oscillatory movement, or would perform with error correction, requiring oscillatory behaviour with small adjustments. Performing with phase resetting is more supportive of an 'interval' type model of timing, as it would require resetting a time keeper at each tone heard. The data therefore suggest some flexibility in these two approaches, and this may be explained by the use of irregular stimuli in the current study, which require alternative mechanisms to those used when engaging with regular stimuli.

Simple modelling of asynchronies using autocorrelation alone demonstrated that for the majority of participants, two autoregressive components provided a better model of the data than just one. This has previously been shown with on-beat tapping (Michon, 1967; Pressing, 1998), but is often overlooked in experiments of this kind when assessing the amount of error correction that might occur. If error correction is only estimated using one asynchrony on the following tap time then that role is likely to be under or over estimated if there is also a significant influence of the asynchrony before. Statistically, 
analysing the data without taking into account all relevant autocorrelation is problematic because it leads to violations of the assumption of independence of the data points. Ideally, autocorrelation should be assessed in all sensorimotor synchronisation experiments, in order to determine how the data should best be analysed.

In the current study, although there was a difference in the relationship between asynchronies and IOIs at different levels of anisochrony, this relationship was not significant in the time series analysis, and was not influenced by whether the anisochrony is perceptible or not. Madison and Merker (2002) generated anisochrony similarly to the current paper and demonstrated that for non-musicians, anisochrony becomes undetectable at rates below $\sim 4 \%$, and sequences with anisochrony greater than $\sim 8.5 \%$ are not attributed with a pulse at all. The results shown in Figure 4 do not suggest any clear differences in performance associated with these thresholds, and this may be a consequence of the structure of the experiment, which involved changes in anisochrony throughout the trial meaning initial perception of anisochrony could establish how people behaved throughout the rest of the trial. None of the time series analysis results suggested that the level of anisochrony changed people's performance.

The current results suggest that people tend not to maintain a steady beat when tapping offbeat with an unsteady stimulus sequence, and are influenced by the preceding stimulus interval in some way. Performance does vary however, and people are able to display some degree of flexibility of approach in this kind of task. This result is primarily significant for our understanding of an "internal timekeeper" used to explain how synchronisation can occur with a beat. In the current case it seems that this internal 
timekeeper was often influenced by an external auditory source, as the majority of people did not maintain a regular pulse while responding to anisochronous stimuli. 


\section{References}

Amazeen, P., Schmidt, R., \& Turvey, M. (1995). Frequency detuning of the phase entrainment dynamics of visually coupled rhythmic movements. Biological Cybernetics, 72(6), 511-518.

Chen, J. L., Penhune, V. B., \& Zatorre, R. J. (2008). Moving on time: brain network for auditory-motor synchronization is modulated by rhythm complexity and musical training. Journal of Cognitive Neuroscience, 20(2), 226-239.

Chen, Y., Ding, M., \& Kelso, S. J. A. (2001). Origins of timing errors in human sensorimotor coordination. Journal of Motor Behavior, 33(1), 3-8.

Delignières, D., Torre, K., \& Lemoine, L. (2009). Long-range correlation in synchronization and syncopation tapping: a linear phase correction model. PloS One, 4(11), e7822.

Drake, C., Penel, A., \& Bigand, E. (2000). Tapping in time with mechanically and expressively performed music. Music Perception, 18(1), 1-23.

Fraisse, P., \& Ehrlich, S. (1955). Note sur la possibilitè de syncoper en fonction du tempo d'une cadence. L'annèe psychologique, 55(1), 61-65.

Gelman, A., \& Hill, J. (2007). Data analysis using regression and multilevel/hierarchical models. Cambridge: Cambridge University Press.

Hary, D., \& Moore, G. (1987). Synchronizing human movement with an external clock source. Biological Cybernetics, 56(5), 305-311.

Huber, P. J. (1964). Robust estimation of a location parameter. The Annals of Mathematical Statistics, 35(1), 73-101.

Kato, M., \& Konishi, Y. (2006). Auditory dominance in the error correction process: A synchronized tapping study. Brain Research, 1084, 115-122.

Keller, P. E., \& Repp, B. H. (2005). Staying offbeat: Sensorimotor syncopation with structured and unstructured auditory sequences. Psychological Research, 69(4), 292-309.

Keller, P. E., \& Repp, B. H. (2005). Staying offbeat: sensorimotor syncopation with structured and unstructured auditory sequences. Psychological Research-

Psychologische Forschung, 69(4), 292-309.

Kolakoski, W., \& Ucoluk, N. (1966). Self generating runs, problem 5304. The American Mathematical Monthly, 73(6), 681-682.

Large, E. W. (2000). On synchronizing movements to music. Human Movement Science, 19(4), 527-566.

Large, E. W., \& Jones, M. R. (1999). The dynamics of attending: how people track timevarying events. Psychological Review, 106(1), 119-159.

Large, E. W., \& Snyder, J. S. (2009). Pulse and meter as neural resonance. Annals of the New York Academy of Sciences, 1169(1), 46-57.

Launay, J., Dean, R. T., \& Bailes, F. (2013). Synchronization can influence trust following virtual interaction. Experimental Psychology, 60(1), 53-63.

Madison, G., \& Merker, B. (2002). On the limits of anisochrony in pulse attribution. Psychological Research/Psychologische Forschung, 66(3), 201-207.

Madison, G., \& Merker, B. (2004). Human sensorimotor tracking of continuous subliminal deviations from isochrony. Neuroscience Letters, 370(1), 69-73. 
Mates, J. (1994a). A model of synchronization of motor acts to a stimulus sequence. I. Timing and error corrections. Biological Cybernetics, 70(5), 463.

Mates, J. (1994b). A model of synchronization of motor acts to a stimulus sequence. I. Timing and error corrections. Biological Cybernetics, 70(5), 463-473.

Michon, J. (1967). Timing in temporal tracking: Van Gorcum Assen, The Netherlands.

Michon, J., \& Van der Valk, N. (1967). A dynamic model of timing behavior. Acta Psychologica, 27, 204-212.

Pikovsky, A., Rosenblum, M., \& Kurths, J. (2001). Synchronization: a universal concept in nonlinear sciences. Cambridge: Cambridge University Press.

Pressing, J. (1998). Error Correction Processes in Temporal Pattern Production: 1. Journal of Mathematical Psychology, 42(1), 63-101.

Repp, B. H. (2000). Compensation for subliminal timing perturbations in perceptualmotor synchronization. Psychological Research, 63(2), 106-128.

Repp, B. H. (2001). Phase correction, phase resetting, and phase shifts after subliminal timing perturbations in sensorimotor synchronization. Journal of Experimental Psychology: Human Perception and Performance, 27(3), 600-621.

Repp, B. H. (2002a). Automaticity and voluntary control of phase correction following event onset shifts in sensorimotor synchronization. Journal of Experimental Psychology: Human Perception and Performance, 28(2), 410-430.

Repp, B. H. (2002b). Phase correction in sensorimotor synchronization: nonlinearities in voluntary and involuntary responses to perturbations. Human Movement Science, 21(1), 1-37.

Repp, B. H. (2004). Comments on "Rapid Motor Adaptations to Subliminal Frequency Shifts During Syncopated Rhythmic Sensorimotor Synchronization" by Michael H. Thaut and Gary P. Kenyon (Human Movement Science 22 (2003), 321-338). Human Movement Science, 23, 61-77.

Repp, B. H. (2005a). Rate limits of on-beat and off-beat tapping with simple auditory rhythms: 1. Qualitative observations. Music Perception, 22(3), 479-496.

Repp, B. H. (2005b). Sensorimotor synchronization: a review of the tapping literature. Psychonomic Bulletin \& Review, 12(6), 969-992.

Repp, B. H. (2006). Does an auditory distractor sequence affect self-paced tapping? Acta Psychologica, 121, 81-107.

Repp, B. H. (2010). Sensorimotor synchronization and perception of timing: effects of music training and task experience. Human Movement Science, 29(2), 200-213.

Repp, B. H., Keller, P. E., \& Jacoby, N. (2012). Quantifying phase correction in sensorimotor synchronization: empirical comparison of three paradigms. Acta Psychologica, 139(2), 281-290.

Repp, B. H., \& Penel, A. (2004). Rhythmic movement is attracted more strongly to auditory than to visual rhythms. Psychological Research, 68, 252-270.

Said, S. E., \& Dickey, D. A. (1984). Testing for unit roots in autoregressive-moving average models of unknown order. Biometrika, 71(3), 599-607.

Schmidt, R. C., \& O'Brien, B. (1997). Evaluating the dynamics of unintended interpersonal coordination. Ecological Psychology, 9(3), 189-206.

Vorberg, D., \& Schulze, H. H. (2002). Linear Phase-Correction in Synchronization: Predictions, Parameter Estimation, and Simulations. Journal of Mathematical Psychology, 46(1), 56-87. 
Vorberg, D., \& Wing, A. (1996). Modeling variability and dependence in timing. In H. Heuer \& S. W. Keele (Eds.), Handbook of perception and action (Vol. 2, pp. 181262). London: Academic Press.

Vos, P., \& Helsper, E. (1992). Tracking simple rhythms: on-beat versus off-beat performance. Time, action, and cognition: Towards bridging the gap, 287-299. 
Table 1

Autoregressive Coefficients in Best Simple Time Series Analysis Model of Asynchrony for Each Participant

\begin{tabular}{lllllll}
\hline Condition & & AR1 & AR2 & AR3 & AR4 & AR5 \\
\hline $\begin{array}{l}\text { Decreasing } \\
\text { Anisochrony }\end{array}$ & Coefficient $M$ & -0.56 & -0.51 & -0.44 & -0.34 & -0.29 \\
& Coefficient $S D$ & 0.25 & 0.25 & 0.18 & 0.10 & NA \\
& $N$ & 28 & 19 & 9 & 5 & 1 \\
\hline $\begin{array}{l}\text { Increasing } \\
\text { Anisochrony }\end{array}$ & Coefficient $M$ & -0.68 & -0.50 & -0.40 & -0.36 & -0.29 \\
& Coefficient $S D$ & 0.20 & 0.18 & 0.15 & 0.13 & 0.01 \\
& $N$ & 33 & 30 & 11 & 4 & 2 \\
\hline
\end{tabular}

Table 1. Autoregressive coefficients in best simple time series analysis model of asynchrony for each participant. Each trial was analysed independently to determine the optimal number of autoregressive components required to model the data, and summary statistics for the relevant coefficients are given here. 
Table 2

Number of Participants in each Strategy Group for each Condition

\begin{tabular}{llll}
\hline Strategy & $\begin{array}{l}\text { Decreasing } \\
\text { Anisochrony }\end{array}$ & $\begin{array}{l}\text { Increasing } \\
\text { Anisochrony }\end{array}$ & Agreement \\
\hline Regular pulse & 13 & 6 & 2 \\
Error correction & 8 & 4 & 0 \\
Phase resetting & 7 & 9 & 5 \\
Negative correction & 4 & 13 & 2 \\
\hline
\end{tabular}

Table 2. Number of participants in each strategy group for each condition.

Agreement indicates the number of participants who used the same strategy in both conditions. 
Table 3

Coefficients of Predictors in Grouped Time Series Analysis

\begin{tabular}{|c|c|c|c|c|c|c|c|c|}
\hline Trial type & Strategy & AR1 & AR2 & AR3 & IOI & $\begin{array}{l}\text { Doubl } \\
\text { etap }\end{array}$ & $\begin{array}{l}\text { Tone } \\
\text { Miss }\end{array}$ & $\begin{array}{l}\text { Nummis } \\
\mathrm{S}\end{array}$ \\
\hline \multirow{4}{*}{$\begin{array}{l}\text { Decreasin } \\
\text { g } \\
\text { Anisochro } \\
\text { ny }\end{array}$} & Regular & 0.00 & 0.20 & 0.18 & $\begin{array}{l}-0.18 \\
*\end{array}$ & $\begin{array}{l}525.1 \\
* *\end{array}$ & $\begin{array}{l}-387.2 \\
* *\end{array}$ & $\begin{array}{l}78.1 \\
* *\end{array}$ \\
\hline & ErrorCorr & -0.27 & -0.15 & -0.10 & $\begin{array}{l}-0.32 \\
* *\end{array}$ & $\begin{array}{l}84 \\
* *\end{array}$ & $\begin{array}{l}-405.1 \\
* *\end{array}$ & $\begin{array}{l}124.5 \\
* *\end{array}$ \\
\hline & PhaseReset & -0.66 & -0.29 & -0.13 & $\begin{array}{l}-0.19 \\
* *\end{array}$ & 10.3 & $\begin{array}{l}-45.4 \\
* *\end{array}$ & $\begin{array}{l}9.5 \\
*\end{array}$ \\
\hline & NegCorr & 0.18 & 0.24 & 0.16 & -0.12 & $\begin{array}{l}548.0 \\
* *\end{array}$ & $\begin{array}{l}-617.1 \\
* *\end{array}$ & $\begin{array}{l}190.1 \\
* *\end{array}$ \\
\hline \multirow{4}{*}{$\begin{array}{l}\text { Increasing } \\
\text { Anisochro } \\
\text { ny }\end{array}$} & Regular & -0.04 & 0.08 & 0.05 & -0.29 & 442.1 & -714.3 & 261.3 \\
\hline & ErrorCorr & -0.07 & 0.08 & 0.15 & $\begin{array}{l}-0.25 \\
* *\end{array}$ & $\begin{array}{l}524.2 \\
* *\end{array}$ & $\begin{array}{l}-696.8 \\
* *\end{array}$ & $\begin{array}{l}364.3 \\
* *\end{array}$ \\
\hline & PhaseReset & -0.58 & -0.24 & -0.02 & $\begin{array}{l}-0.23 \\
* *\end{array}$ & $\begin{array}{l}240.4 \\
* *\end{array}$ & $\begin{array}{l}- \\
178.1 \\
* *\end{array}$ & $\begin{array}{l}53.7 \\
* *\end{array}$ \\
\hline & NegCorr & -0.13 & 0.04 & 0.11 & $\begin{array}{l}-0.26 \\
* *\end{array}$ & $\begin{array}{l}431.2 \\
* *\end{array}$ & $\begin{array}{l}-551.6 \\
* *\end{array}$ & $\begin{array}{l}177.9 \\
* *\end{array}$ \\
\hline
\end{tabular}

Table 3. Coefficients of predictors in grouped time series analysis. Strategy is given by 'Regular' (regular pulse), 'ErrorCorr' (typical error correction), 'PhaseReset' (phase resetting) or 'NegCorr' (negative error correction). ** indicates $\mathrm{p}<0.01$, * indicates $\mathrm{p}<$ 0.05, significance is not calculated for AR components. 
OFF-BEAT TAPPING WITH ANISOCHRONY

42

Figure 1. Methods of off-beat tapping. IOI line gives the stimulus interonset intervals, and the responses expected in the case of each of the three proposed methods of tapping are given above. Circles represent events (either taps or tones). Larger numbers give ITI or LOIs (for example in milliseconds) and smaller numbers give asynchronies between tone and tap times.

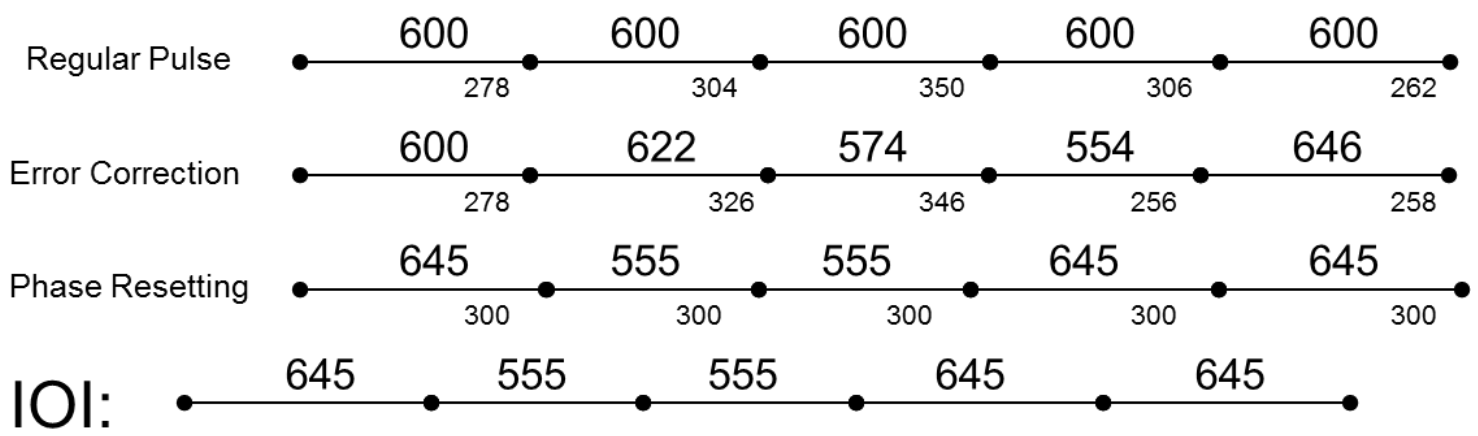


Figure 2. Histograms of raw tapping data. Asynchrony and ITI raw data are given for each of the two conditions.
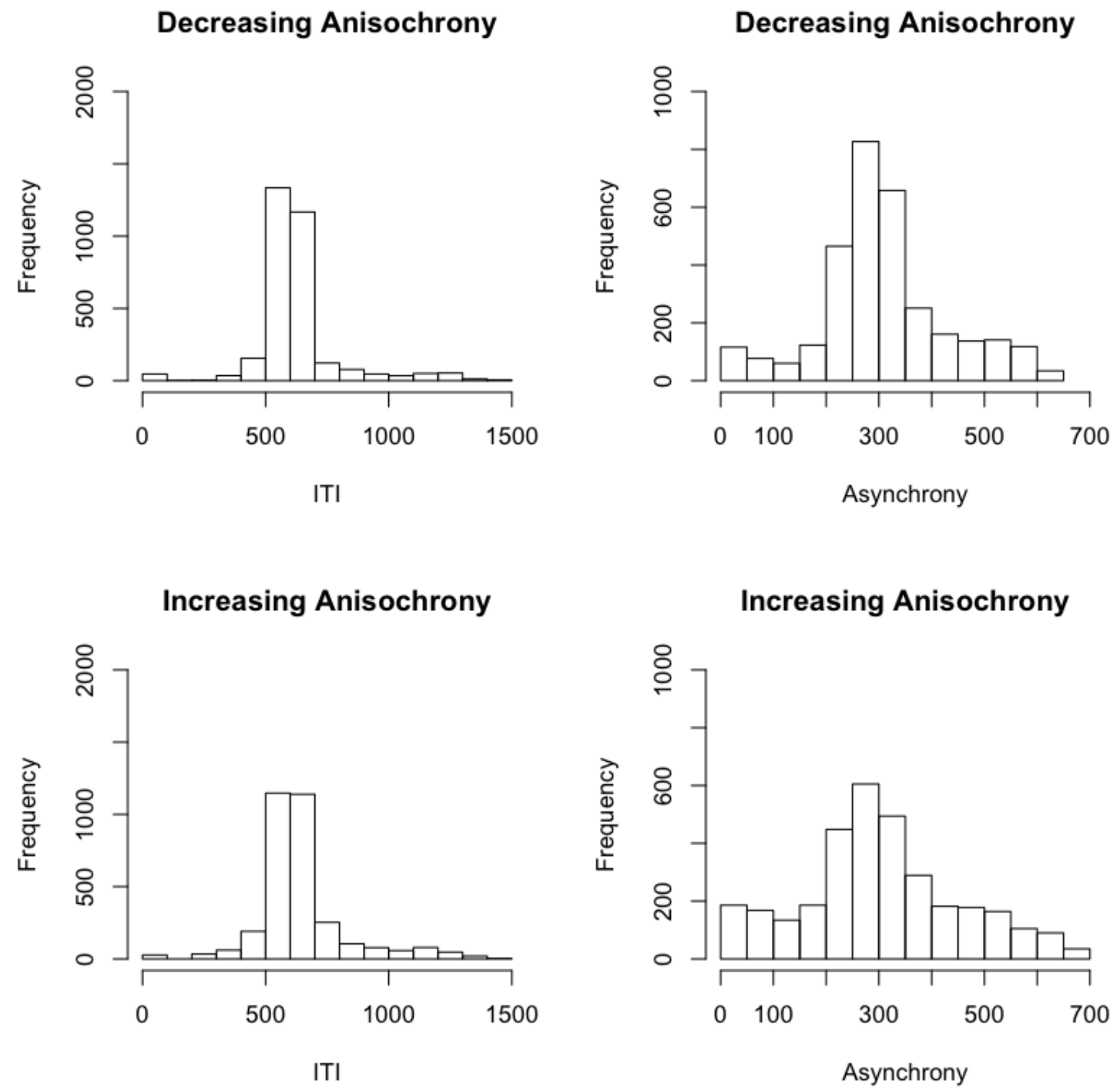
Figure 3. Relationship between IOI and measures of ITI and asynchrony. The amount of deviation from $600 \mathrm{~ms}$ in IOI is plotted against the deviation in participant responses in the following ITI and asynchrony. Bars indicate standard error.
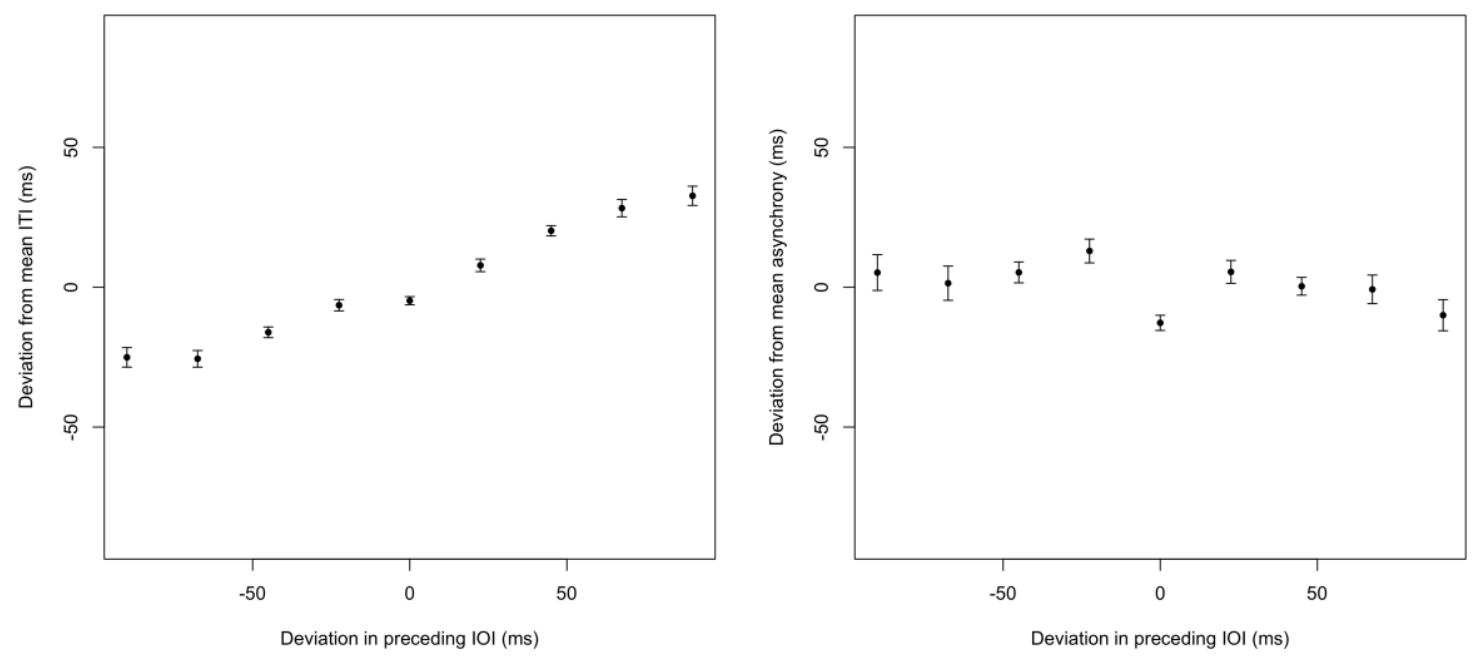
Figure 4. Relationship between level of anisochrony and influence of IOI on measures of ITI and asynchrony. Here, the relationship between IOI and each dependent measure has been recalculated for each level of anisochrony. Points indicate the coefficient of these linear relationships, with bars giving the standard error.
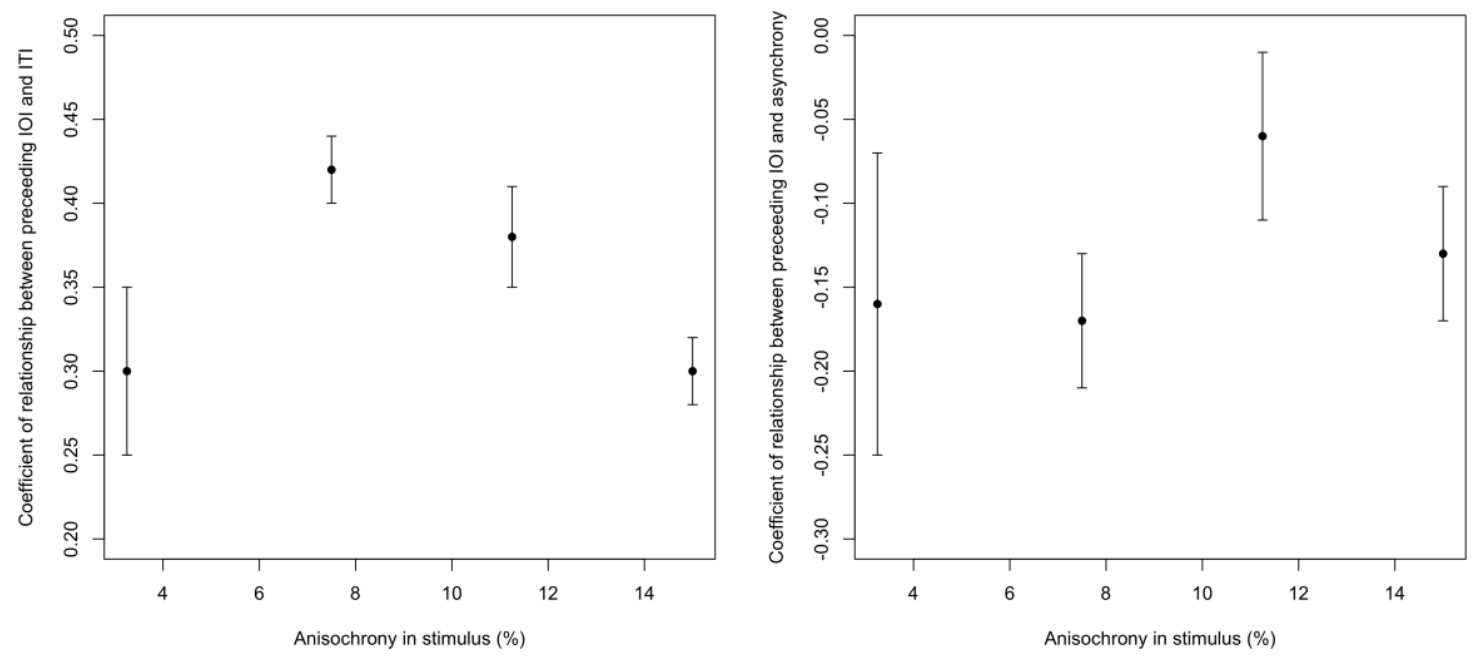
Figure 5. Examples of individual tapping trials. Each panel gives raw ITI data for one participant analysed using full time series analysis. ITI data is plotted against the tap number in each trial (so that each panel represents a time series from the start to the finish of one tapping trial going from left to right). IOI data is plotted in grey and as ITIs have been matched with previous IOIs, points appearing at the same point on the graph actually represent an IOI and the following ITI. Note that the y-axis has a different scale in the middle panel because this participant missed many taps. 

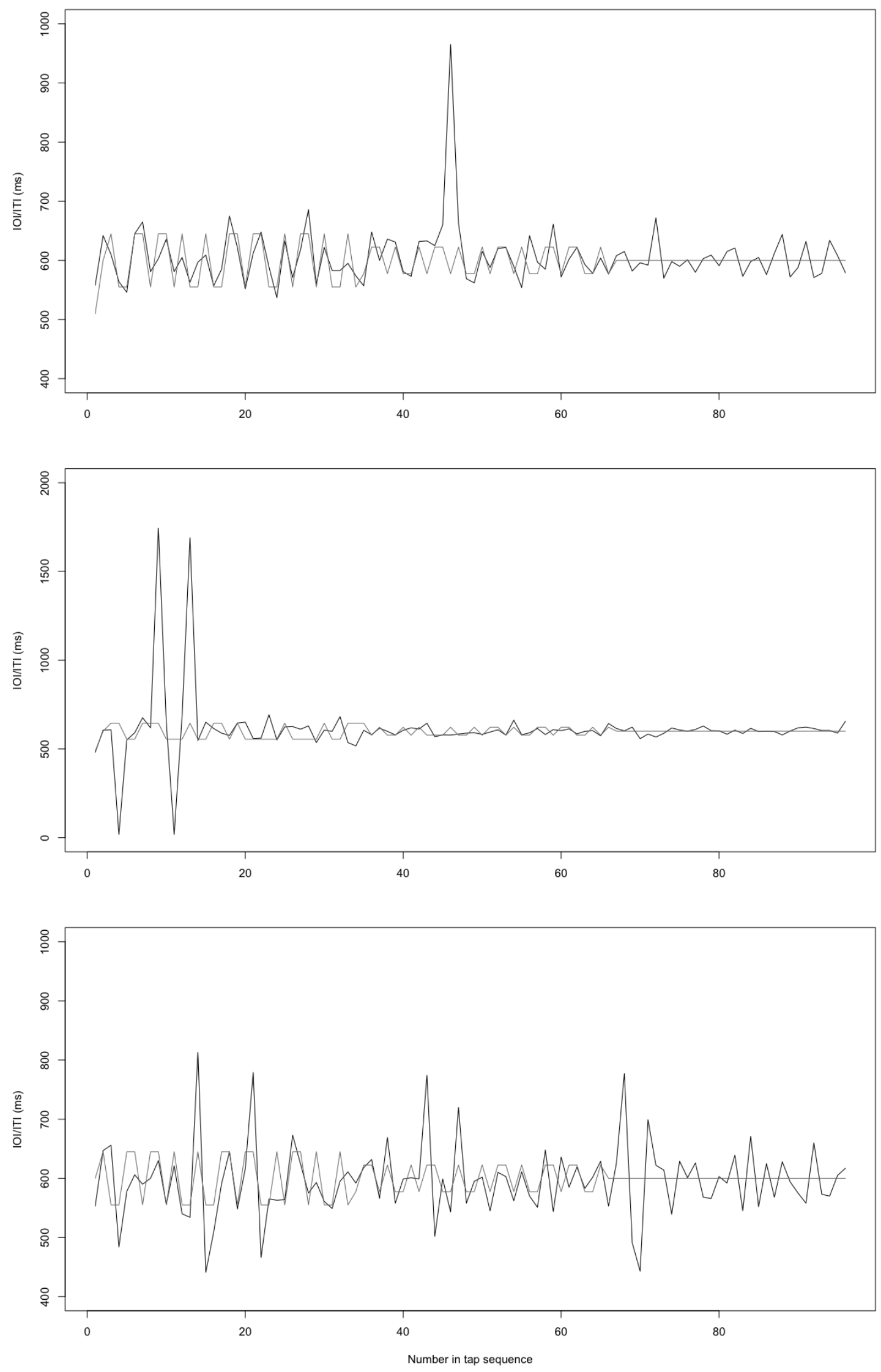